\title{
CONFIGURACIÓN DE ESPACIOS DEY PARA LA VIDA A PARTIR DE LAS RELACIONES CON EL AMBIENTE QUE ESTABLECEN LAS MUJERES DEL MOVIMIENTO POPULAR DE MUJERES LA SUREÑA
}

\section{Configuration of Spaces for Life, Based on the Relations with the Environment Established by the Members of the Women's Popular Movement, La Sureña}

\section{Configuração de espaços de e para a vida a partir das relações com o ambiente que estabelecem as mulheres do movimento popular de mulheres La sureña}

Fecha de recepción: 29 de marzo 2016

Fecha de aceptación: 1 de junio de 2017

\section{Leidy Lorena Ramírez Rodríguez ${ }^{1}$}

\section{Resumen}

Este artículo presenta los resultados del "Proyecto educativo no formal de mujeres La Sureña. Construyendo una sociedad igualitaria: configuración de espacios de y para la vida desde y para la mujer", realizado durante la práctica pedagógica dentro de la Licenciatura en Biología de la Universidad Pedagógica Nacional.

Este se llevó a cabo con el Movimiento Popular de Mujeres La Sureña, en la localidad de Bosa, con las mujeres de los grupos 2-3 (quinto, sexto y séptimo grados) en el componente biocultural (correspondiente a Biología y Ciencias Naturales), cuyo objetivo fue analizar las relaciones que establecen las mujeres con el ambiente y cómo lo configuran. A partir de las clases y talleres, se analizaron las concepciones de las mujeres acerca del ambiente y las relaciones que establecen con este, configurando los espacios en los cuales están inmersas.

Con respecto a la metodología, se encuentran las clases y talleres que se realizaron a partir del diálogo de saberes realizados con las mujeres, el análisis, sistematización y reflexión acerca de estos, la pedagogía crítica y el enfoque sociocrítico. Para esto, el análisis correspondiente se expondrá en cinco fases principales y de acuerdo con estas se obtuvo como resultado que las mujeres no conciben el ambiente como ajeno a ellas, aunque se hace énfasis en prácticas de cuidado del mismo y con otros organismos; además, se comprende, de acuerdo con lo anterior y como resultados, las siguientes relaciones: Mujer-Mujer, Mujer-Familia, MujerTrabajo, Mujer-proceso (La Sureña), Mujer-Ambiente, Mujer-Vida y Mujer-Vivo, las cuales serán desglosadas dentro del artículo.

1 Estudiante de Licenciatura en Biología, décimo semestre, Universidad Pedagógica Nacional de Colombia. 
De este modo, la práctica pedagógica y el trabajo realizado con las mujeres permite evidenciar toda una serie de concepciones del ambiente y cómo estas lo configuran, como mujeres y sujetas activas en la sociedad, el rol que cumplen en la misma, sus sentires, saberes y su empoderamiento.

Palabras clave: Ambiente; educación popular feminista; pedagogírítica; contexto; reconocimiento; enfoque de género.

\section{Abstract}

This paper presents the results of the "Non-formal educational project for women of La Sureña. Building an egalitarian society: configuration of spaces for life, from and for women," which was carried out during the teaching practice of the Degree in Biology of Universidad Pedagógica Nacional.

This project was carried with the women's popular movement of La sureña in the locality of Bosa with the women from groups 2-3 (fifth, sixth, and seventh grade) in the biocultural component (i.e., Biology and Natural Sciences), with the purpose of analyzing the relationships that women establish with the environment and how they do it. Based the classes and workshops, we analyzed the women's conception of the environment and their relationship with it, thus configuring the spaces in which they are immersed.

As for the methodology, there are the classes and workshops given based on the dialogue between the women, the analysis, systematization and reflection on them, critical pedagogy and the socio-critical approach. For this purpose, the corresponding analysis will be explained in five phases, based on which it was found that women do not perceive the environment as being alien to them, although emphasis is made on practices related to the care of the environment and other organisms; based on this, and as a result, the following relationships are found: Woman-Woman, Woman-Family, Woman-Work, Woman-Process (La Sureña), Woman-Environment, Woman-Life, and Woman-Living, which will be explained in detail in the paper.

Thus, the pedagogical practice and work carried out with the women reveals a series of conceptions about the environment and how they shape it, as women and active individuals of society, their role in it, their feelings, knowledge, and empowerment.

Keywords: Environment; feminist popular education; life; critical pedagogy; context; recognition; gender approach

\section{Resumo}

Este artigo resultado os resultados do "Projeto educativo não formal de mulheres La Sureña. Construindo uma sociedade igualitária: configuração de espaços de e para a vida desde e para a mulher”, realizado durante o estágio dentro da Licenciatura em Biologia da Universidade Pedagógica Nacional.

O projeto realizou-se com movimento popular de mulheres La Sureña, na localidade de Bosa, com as mulheres dos grupos 2-3 (quinto sexto e sétimo ano) no componente biocultural (correspondente a Biologia e Ciências Naturais), cujo objetivo foi analisar as relações que estabelecem as mulheres com o ambiente e como este é configurado. A partir das aulas e oficinas, analisaram-se as concepções das mulheres acerca do ambiente e as relações que estabelecem com ele, configurando os espaços nos que estão imersas.

Com relação à metodologia, encontramos as aulas e oficinas realizadas a partir do diálogo de saberes com as mulheres, a análise, sistematização e reflexão sobre esta, a pedagogia crítica e a abordagem sociocrítica. Para isso, a análise correspondente será exposta em cinco fases principais nas que evidenciou-se como resultado que as mulheres não concebem o ambiente como algo afastado delas, ainda que se faz ênfase nas práticas de cuidado deste e com outros entes. Além disso, são levadas em conta, de acordo com o anterior e como resultados, as seguintes relações que serão desglosadas no artigo: Mulher-Mulher, Mulher-Família, Mulher-Trabalho, Mulher-processo (La Sureña), Mulher-Ambente, Mulher-Vida e Mulher-Vivo.

Dessa forma, a prática pedagógica e o trabalho realizado com as mulheres permite evidenciar toda uma série de concepções do ambiente e como elas o configuram, como mulheres e sujeitos ativos na sociedade, o papel que têm nela, seu sentir, seus saberes e seu empoderamento.

Palavras-chave: ambiente; educação popular feminista; vida; pedagogia crítica; contexto; reconhecimento; abordagem de gênero 


\section{Una oportunidad para las mujeres de Bosa}

En nuestro país se trabaja para generar propuestas y posibles soluciones para mejorar la calidad de la educación formal, la cual no está diseñada para cierto tipo de población que por motivos como la edad, condiciones socioeconómicas o culturales, violencia, desplazamiento, desigualdad, entre otros, tienen un nivel educativo mínimo o nulo. A pesar de esto, no se han logrado resultados contundentes, considerando que la educación colombiana concibe todo el territorio como uno solo: el contexto, formas de vida y de aprendizaje como homogéneos, que no cambian ni se configuran por distintos factores, condicionando las dinámicas de la sociedad e incidiendo en la pérdida de muchas nociones, costumbres, saberes, formas de comprender el mundo y la configuración a partir de la tradición y el contexto, el cual, entre otros factores, lo conforman personas de diferentes edades.

Por otro lado, el sistema educativo actual hace que todos sus miembros sean víctimas de discriminación y desigualdad, así lo afirma Gonzalo Cataño (s.f.) en su escrito Educación y Diferenciación Social en Colombia:

La estratificación social toma en sus manos el sistema educativo y trata de moldearlo a su imagen y semejanza. Ello hace que el prestigio y rango de las instituciones que conforman el sistema educativo estén estrechamente asociados a la posición social ocupada por las familias de sus estudiantes [...]. Las especialidades que ofrece el sistema educativo también están marcadas por la estratificación social”. (p. 3).

Así, el acceso a la educación, la calidad y las dinámicas educativas no atienden las necesidades de todos los sectores socioeconómicos y favorecen los intereses de algunas minorías. De esta manera se han formado grupos populares fundamentados en el derecho a la educación con población vulnerada educativamente que no cuenta con la posibilidad de acceder a ninguna forma de educación, entidades en su mayoría sin ánimo de lucro, las cuales aportan a la alfabetización y fortalecimiento educativo y personal de las personas desde perspectivas heterogéneas de acuerdo a las necesidades.

En la localidad de Bosa se encuentra el proceso Movimiento Popular de Mujeres La Sureña, que nace "de la confluencia de distintas iniciativas colectivas e individualmente que vienen realizando trabajos académicos, políticos, artísticos, y productivos con mujeres y desde mujeres de sectores populares, enfocado en la identidad entendida como un proceso de construcción social y cotidiana que articula diferentes formas de entender, asumir y relacionarse con el mundo, articulada a la perspectiva de trabajo desde el género, la clase, la generación y la etnia (Torres, González y Pérez, 2012). Este proceso se constituyó en articulación con la Licenciatura en Educación Comunitaria de la Universidad Pedagógica Nacional con énfasis en Derechos Humanos, como una propuesta de educación de mujeres adultas con enfoque de género elaborada por una docente de la Universidad Pedagógica Nacional y dos estudiantes de la Licenciatura en Educación Comunitaria en la Universidad Pedagógica Nacional, como las coordinadoras del proceso desde el año 2012. Es así como las mujeres encuentran la oportunidad de tener una posibilidad educativa sin importar su condición económica, social, educativa, generacional $y$, además, se enfatiza en que dichas mujeres construyan una identidad frente a la sociedad y el papel que ocupan en la misma.

El proceso de enseñanza y aprendizaje y la construcción colectiva de saberes con las mujeres se dio a partir de sus sentires y su diversidad como sujetas, su visión acerca del mundo de la vida y lo vivo y las relaciones que establecen con estos, lo cual hace que se configuren y reconozcan a sí mismas. Es importante mencionar que se trabaja a partir de cuatro componentes: pensamiento lógico y matemático, comunicación enfocada a la comprensión de lectura, sociocultura y biocultura, teniendo en cuenta el enfoque de género como transversal a cada componente.

De acuerdo con lo anterior, para desarrollar la práctica pedagógica, como estudiante de la Licenciatura en Biología, se propuso la siguiente temática con el fin de visibilizar los espacios de interacción y empoderamiento del papel de la mujer en la sociedad desde la experiencia y el diálogo de saberes: ¿Cuáles son las relaciones que establecen las mujeres con su ambiente por medio de las cuales configuran a sí mismas y a este como espacios de y para la vida, a partir de las concepciones de las mujeres y el conocimiento construido?

La importancia de este trabajo radica principalmente en que no se ha abordado el ambiente desde una perspectiva distinta con mujeres desde la educación popular feminista y no hay autores que hablen desde estos aspectos particulares. Además, hay tener en cuenta que es necesario concebir el maestro de Biología de otras formas y desde otros espacios, lo cual enriquece la práctica y la construcción colectiva de saberes.

\section{Metodología}

Para llevar a cabo este trabajo se tomaron en cuenta las siguientes bases pedagógicas e construcción de conocimiento: En primera instancia, la investigación cualitativa 
desde Taylory Bogdan (1986, p. 19) se define como "aquella que produce datos descriptivos: desde las palabras de las personas y la conducta observable"; además, sugieren características como tener una visión holística y que el investigador no reduzca los escenarios y la población a variables sino que se consideren como un todo.

Puede entenderse como una construcción de diseños de investigación a partir de la observación y otros instrumentos de recolección de datos, ya que se considera hay un interés por el contexto del acontecimiento y en vez de ser modificados por el investigador, este tiene un carácter y una responsabilidad social, y se centra en los sujetos que vivencian todo tiempo acontecimientos y acciones en su diario vivir.

Además, el paradigma utilizado para esta investigación fue el paradigma sociocrítico que tiene como propósito no solo la comprensión, interpretación y reflexión, sino además la transformación de la realidad estudiada con el fin de resaltar el papel del diálogo y la necesidad de la interpretación. Así, se orienta a la acción, es una investigación-acción participativa, transformadora con respecto al objeto. Se trata de una investigación en la educación, que "[...] se caracteriza por incorporar criterios históricos, contextuales y valorativos en la construcción del conocimiento que se produce en y para la acción" [...] "mediante una visión participativa, dialógica y holística del conocimiento, donde el sujeto es el elemento principal evaluando sus acciones transformadoras y su autotransformación" (Colunga, García y Blanco, 2013, p. 17).

Todo esto fue complementado con el modelo de la pedagogía crítica, que según Brito (2008) significa un punto de partida para problematizar y convertir la educación en una herramienta al servicio del cambio y la transformación de las sociedades. Asimismo, se tiene en cuenta lo planteado por Paulo Freire, quien habla de la función crítica como proceso que debe nutrirse de la realidad del presente en pro de reconstruir continuamente los métodos educativos articulados a la práctica pedagógica que la pedagogía crítica demanda.

Dentro de la metodología se encuentra también como parte fundamental el cuaderno de campo, las entrevistas, talleres y los aspectos éticos pertinentes donde las mujeres que participan del proceso conocen de la realización y postulación de este escrito y su coordinadora.

\section{¿Cómo abordar la enseñanza de la Biología desde el Movimiento Popular de Mujeres la Sureña?}

Para llevar a cabo la práctica pedagógica, se tuvieron en cuenta enfoques, bases y dinámicas del proceso de La Sureña al trabajar con y para las mujeres. Entre los aspectos más destacados se encuentran:

- La educación popular abordada por Brito, De Lourdes y Maribal (2008). Consiste en un proceso desde una perspectiva que se nutra de la realidad del presente para reconstruir continuamente los métodos educativos articulados en la práctica educativa para la transición social articulada a una conciencia de participación popular, la cual es una propuesta de redefinición de los actores sociales y sus funciones, que rompe con las formas tradicionales de educación (con la estructura y la institucionalidad establecida), así como también impone una nueva distribución del saber del pueblo y del acceso público y participativo de la educación.

- La educación popular feminista desde Sáenz y Rapacci (2013) se entiende como pensamiento y campo de movilización política, que impulsa a las organizaciones populares de mujeres a buscar nuevas explicaciones acerca de las situaciones de opresión que afectan a las mujeres en su diversidad cultural y social. Se afecta también a nuevas formas de ejercicio político acordes con las condiciones particulares de participación femenina, dada la división sexual del trabajo y roles de género impuestos por la cultura del patriarcado. Este proceso favorece la construcción de las mujeres como sujetos políticos, al acompañar, valorary aprender colectivamente sobre su experiencia.

Así, reflexionar sobre estos puntos es necesario para debatir sobre el paradigma de la pedagogía del oprimido, de la educación popular, de la pedagogía critica en los espacios de formación que proveemos; generar espacios de formación como de resistencia y de construcción de mundo; inscribir los procesos de formación de técnicos y técnicas del campo en un proyecto mayor transformador, emancipador, liberador, teniendo como principio el saber acumulado por los participantes.

En ese sentido, este proceso se llevó a cabo con un grupo de trabajo 2-3 que quedó conformado por 14 mujeres que están entre las edades de 16 y 60 años aproximadamente, lo cual indica que es un grupo bastante heterogéneo ya que hay varias mujeres cercanas a este rango de edad. 
Ahora bien, dentro de la interacción con las mujeres además de las clases realizadas en el componente de Biocultura, se trabajaron talleres por medio de los cuales se abordó e indagó acerca de sus concepciones de ambiente y sus relaciones con este, en los cuales las mujeres fueron muy participativas, respetaron y compartieron sus opiniones y reconocieron la diversidad de formas de concebir el mundo.

\section{Implementación a partir del acercamiento y diálogo de saberes con las mujeres}

El diálogo de saberes visto desde Urbina (2013) se ha venido entendiendo como el ejercicio

profundamente humano de intercambiar ideas o pensamientos en forma pacífica, a partir de lo fundamental de cada dialogante quien aparece como un vocero y/o representante de la cultura respectiva. Estos diálogos interculturales se desarrollan en orden a lograr un buen manejo de ese Mundo que es la sumatoria de todos los mundos (los particulares de cada cultura). El «buen manejo de mundo» que busca este diálogo, tiene como fundamento el respeto y como meta lograr un «estado de justicia» en que todo y todos quepamos fraternalmente. (p. 1).

\section{Fase 1. Me reconozco y reconozco mi ambiente}

Se encontró que, desde el diálogo con las mujeres, no todas las personas somos iguales y que desde la diferencia se pueden construir conocimientos y sociedad. Además, con el grupo se definió que el concepto "ambiente" no es solo la naturaleza sino también todo lo que nos rodea y con el cual establecemos relaciones.

Luego, en forma de escrito, las mujeres respondieron a las preguntas: ¿Quién Soy?, ¿cómo me reconozco?, ¿qué papel desempeño en la sociedad?, ¿cómo me relaciono con mi ambiente?

Las mujeres expresaron durante el ejercicio de escritura que se sentían a gusto pensando en sí mismas y concebirse importantes dentro de la sociedad, al socializar los escritos las mujeres que escucharon atentas; de hecho coincidieron en varios de los aspectos que se tomaron en cuenta. Por esto, de acuerdo a la actividad, surgieron varias categorías: lugar de origen, la maternidad, sus cualidades, su escolaridad (porque no pudieron culminar sus estudios en la educación formal), cuál es su papel en la sociedad, su relación con el ambiente y sus expectativas dentro del proceso. Esta actividad culminó con un cuento que cada mujer realizo sobre su vida.

\section{Fase 2. ¿Qué es la vida y lo vivo?}

A partir de esta pregunta, las mujeres hablan de la vida desde las emociones y la armonía enfatizando desde la generalidad que "la vida es hermosa, que hay que saberla llevar superando los obstáculos, ayudando a las personas que lo necesitan. Vida es cantar, estudiar, trabajar, bailar, jugar y otras actividades que se pueden hacer, agradecer por la salud y las bendiciones recibidas" (Recopilación de las similitudes y/o perspectivas repetitivas en los escritos de las mujeres).

Para hablar de lo vivo, las mujeres comentaron desde lo que aportan los seres vivos no solo a lo humano sino también al mundo de lo vivo, por ejemplo, "se estabilizan y estabilizan el medio ambiente, los animales y vegetales sirven de alimento" (Testimonio de estudiante). También, se hace relación al humano como ser racional y que puede disfrutar de todo lo vivo, es decir, de los demás organismos y los factores inorgánicos de la naturaleza y cómo podemos cuidar y vivir en armonía o perjudicar a estos y a nosotros mismos.

Desde la visión de una mujer de comunidad, lo vivo es todo lo que existe y con lo que nos relacionamos y somos interdependientes; ella enfatizó mucho en la importancia de las plantas desde la medicina tradicional y cómo estas deben ser cuidadas.

\section{Fase 3. Violencias de género}

Este tema fue propuesto por una de las maestras, donde se habló de la desigualdad de roles no solo a nivel del hogar, sino en lo laboral, económico y político, haciendo énfasis principalmente en el ámbito doméstico y laboral.

Se expusieron, desde los derechos humanos, las violencias contra las mujeres, que son cinco: violencia física, violencia psicológica, violencia económica, violencia patrimonial y violencia sexual. Durante el taller, la mayoría de las mujeres expresan que se han sentido víctimas de alguna de las violencias expuestas y del mismo modo conocen otras mujeres que son víctimas de las mismas, como por ejemplo, no tener derecho a estudiar por ser mujeres, entre otras desigualdades que evidencian en el taller. Se enfatizó en que estas violencias no pueden dejarse pasar por alto, que son un delito, demandables por la víctima o por terceros y que esto puede realizarse de manera anónima. 
Para finalizar, se hablaron de otros derechos que las mujeres han perdido como por ejemplo, vivir en una cultura libre de sexismo, derecho a la educación, a la salud, a vivienda digna, al trabajo remunerado, entre otros, y que son derechos que las mujeres quieren recuperar para cumplir sus metas y sueños que han dejado atrás.

\section{Fase 4. Primer Encuentro de Educación Popular Feminista La Sureña: "Mujeres y Hombres con Experiencias, Testimonios y Apuestas desde el Sur"}

Junto con mujeres de Madrid, Cundinamarca se socializaron las experiencias de educación popular feminista desde los procesos asistentes a través de un panel con varias preguntas, en el cual participaron dos mujeres de otros movimientos populares feministas, una mujer de La Sureña y una invitada que es la hermana de Rosa Elvira Cely, mujer víctima de feminicidio.

Desde la grabación y toma de notas en el cuaderno de campo y su análisis se concluye desde el panel que:

- El feminismo tiene en cuenta a la mujer como sujeta central y sus derechos, por ejemplo, la salud y soberanía alimentaria. No se trabaja con las mujeres desde la vulnerabilidad, sino desde la visibilización, reconociendo su contexto, desde propuestas ético-políticas, buscando recuperar la naturaleza de la mujer en la sociedad y el ambiente natural y cultural.

- La educación popular es un plan de vida, viene de lo ancestral, de la reconstrucción de la historia y la cultura, del territorio, desde la lucha, hermandad y solidaridad en busca de una vida digna. Se trabaja desde la corporalidad, emocionalidad y espiritualidad; así, es importante solicitar y brindar información que aporte a la configuración de las mujeres y estas puedan brindar ayuda a otras que lo requieran. La educación popular feminista es comunitaria (un conjunto de personas encaminadas hacia un único propósito), se comparte el territorio a partir de la diversidad, de vincular varios procesos pero se dirigen desde lo colectivo, buscando la transformación de las mujeres y la sociedad.

Para terminar el encuentro se realizaron actividades de integración y se compartieron testimonios de las mujeres de La Sureña, los cuales fueron muy gratificantes, conmovedores y emotivos. Las mujeres afirmaron que han transformado su vida, su forma de verse y reconocerse ante ellas mismas, sus familias y la sociedad, que son conscientes que deben y merecen ser escuchadas, de sus derechos y deberes, que ya no sienten miedo a expresar sus deseos y opiniones, y finalmente agradecieron al proceso y a todas y todos los que hicieron parte de este.

\section{Fase 5. Taller acerca de la escuela y su relación con la mujer.}

En este taller, una maestra de la Lic. En Educación Comunitaria de la UPN, expuso el concepto de escuela como uno de los principales dispositivos en la constitución de subjetividades femeninas y masculinas, las relaciones de género (poder) desiguales que se van naturalizando e impactan en el autoestima y en la imagen de los sujetos, los procesos de esencialización a partir de las cuales se constituyen juicios morales y cómo la escuela ofrece modelos de identificación en los roles género, clase social, que legitiman percepciones sobre lo masculino y femenino. A partir de esto se ha desplegado un modelo de mujer: dedicadas al hogar, delicadas, que no pueden ser agresivas ni violentas, y del mismo modo se establece un modelo de hombre de igual manera con ciertas características.

Desde la violencia de género en la escuela se habla acerca de las afirmaciones de género que suceden en el ámbito institucional, como por ejemplo, afirmar que los profesores (hombres) son más competentes académicamente que las profesoras; la singularización de los niños y niñas en el aula de clase, el sistema de valores transmitido en espacios académicos diferentes para hombres y mujeres, individual y en género. Por lo tanto, se requiere que las mujeres dejen de tener el papel de moderadoras y se vuelvan voceras y liderezas, dejar de pensar que los hombres son inteligentes y las mujeres delicadas.

Como última medida, se socializaron con las mujeres sucesos que hicieron que se vincularan más a la sociedad, como por ejemplo, el acceso a las mujeres a la educación (hasta cierto nivel y a cierto tipo de saberes, como la costura, bordado, cocina, cuidado de niños, etc.), el acceso de las mujeres a la educación media y a la universidad, y el derecho al voto, aunque algunos de estos derechos hayan tenido un trasfondo y unos intereses aparte de que las mujeres se vincularan a la sociedad.

\section{Sistematización y análisis de la información}

Después del tiempo compartido con las mujeres, la construcción de conocimiento y la información recopilada a partir de las clases del componente biocultural, se realizó una matriz de categorización y de su posterior análisis 
surgieron las siguientes relaciones de las mujeres con su ambiente:

- Relación Mujer-Mujer: Dentro de esta relación las mujeres se conciben como un ambiente, parte de otros ambientes (ya que en su mayoría expresan que estamos y hacemos parte del ambiente como seres vivos que nos relacionamos con el mismo), y por supuesto como territorio, lo que propicia una relación de conocimiento y reconocimiento de sí mismas. Sin embargo, lo más habitual es que al hablar de sí mismas describen su fisonomía y sus cualidades; además, enfatizan bastante en la idea de cómo sus cualidades les permiten desenvolverse de una buena manera en la sociedad, de llevarse bien con los y las que les rodean, y siempre les sea posible ayudar a quien lo necesite.

- Relación Mujer-Familia: Se puede evidenciar que la relación que las mujeres establecen con sus familias y sobre todo con sus hijas e hijos es muy fuerte, puesto que afirman constantemente que desean ampliar su campo de posibilidades dentro de la sociedad para brindarles un mejor ejemplo y futuro. Del mismo modo desean ampliar sus ingresos y tener un buen vivir, mejorar ciertas condiciones y algunas ven que sus hijas e hijos ya no son infantes, desean estudiar y prepararse ahora que tienen el tiempo y la disposición.

Las mujeres también hablan de sus familias, padres y madres, desde la infancia. Algunas contaron con la compañía de su familia nuclear y en ciertos casos esto se mantiene, mientras que otras se criaron con otros familiares pero, en la mayoría de situaciones, su acceso a la educación fue limitada por distintas circunstancias. Por esto, tienen un deseo no solo de enriquecer sus conocimientos y poder tener otros roles dentro de la sociedad, sino que ahora es importante reconocerse dentro de sí mismas, sus familias y la sociedad como iguales, importantes, y que pueden aportar desde distintos ámbitos a partir del empoderamiento que realiza dentro del proceso La Sureña.

Por lo tanto, se reitera la relación de cuidado de las mujeres con sus familias y de hecho, al dedicarse a esta relación que se les ha inculcado desde niñas (ser buenas madres, esposas y dentro de su labor doméstica etc.), es posible afirmar que han dejado de lado su relación consigo mismas; lo cual no quiere decir que esto sea malo ya que las mujeres dentro de la sociedad y sus familias procrean, dan y cuidan la vida, así se hace necesario resaltar esta labor de las mujeres dentro de la sociedad que muchas veces no es valorada y visibilizada, ya que esto y la labor doméstica pueden considerarse como trabajos no remunerados.

- Relación Mujer-Trabajo: Al hablar del ámbito laboral, algunas mujeres no trabajan, ya que se dedican a su hogar como madres y esposas; otras, debido a su edad, no consiguen empleo y en su mayoría trabajan, pero suelen afirmar en repetidas ocasiones que sus trabajos son muy exhaustivos y muy mal remunerados, esto debido principalmente a que su bajo nivel de escolaridad no les permite conseguir empleos en otros sectores diferentes principalmente a las labores domésticas, las ventas, reciclaje, entre otras. Del mismo modo, desde la labor que desempeñen las mujeres en sus lugares de trabajo, buscan establecer relaciones amables y armónicas con quienes les rodean, por lo cual se puede afirmar que buscan ambientes amables para desempeñar sus tareas y por ejemplo, desde la labor en oficios varios y en el hogar, benefician a todos y todas los que residen y acceden a estos lugares por distintos motivos, adquiriendo una relación de cuidado, organización y planeación para que las dinámicas de los lugares donde están inmersas funcionen de la manera más correcta. Además, agradecen al proceso al poderse vincular sin entorpecer otras actividades como el trabajo.

- Relación Mujer-Ambiente: Todas las mujeres del grupo de trabajo coinciden en que el ambiente no debe ser concebido como la naturaleza o lo que comúnmente se denomina "lo verde". Esto no quiere decir que estos espacios no sean ambiente, por supuesto que sí, pero no son el único ambiente que existe, sino que también lo que nos rodea, por ejemplo, los ambientes pueden ser totalmente urbanos, y del mismo modo todo con lo cual y con quienes nos relacionamos.

De esta manera, la principal relación que se puede establecer dentro del ambiente es la posibilidad que brindan las mujeres a la conformación de los mismos y por qué no, a que estos se mantengan, puesto que las mujeres velan por el bienestar de los lugares, ámbitos y relaciones que establecen. Asimismo, las mujeres exponen que hay ambientes que son inseguros o dentro de los cuales no se sienten a gusto o bien recibidas, lo cual muestra que las relaciones que se dan en el ambiente no siempre suelen ser positivas. Así, las mujeres hablan de cómo tratan de comportarse de la forma más correcta posible dentro del ambiente en el que se encuentren, tratan de no afectar las dinámicas del mismo ni de quienes lo conforman o transiten en el mismo. Por lo tanto, se establecen relaciones de respeto por el otro/a, por el territorio (entendido más que como un terreno o lugar) y como el

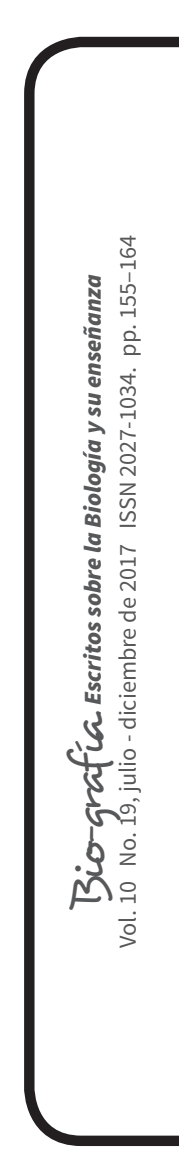


primer territorio o ambiente con que se relacionan es consigo mismas, por la integridad y el buen vivir.

- Relación Mujer-Vida: Al hablar de vida las mujeres en su totalidad hablan con gusto, es decir, no se perciben ni se escuchan por parte de ninguna mujer algún comentario negativo. De acuerdo con esto, la relación de las mujeres con la vida da un sentido al ambiente y viceversa, debido a que todas las actitudes, acciones y actividades que las mujeres manifiestan acerca de la vida propician y configuran el ambiente. Asimismo, las mujeres, al hablar de vida, hacen énfasis sobre lo que hacen o les gustaría hacer, que lo que las hace felices y da sentido a su vida, ya que como ellas indican la vida es ese tránsito a partir de las experiencias nos permite configurar quienes somos, como actuamos y como nos relacionamos (ya que esto no ocurre de la misma manera en todas las personas). En ese sentido, puede decirse que la noción de vida, como transcurre y se concibe, va a incidir en cómo funciona y se desarrolla también el ambiente, y por supuesto al hablar de vida, la mujer o la hembra de cualquier especie dentro del mundo natural tiene la labor elemental de enseñary mostrar a sus hijos el ambiente y las dinámicas donde están inmersos para que esto no sea entorpecido, es decir, la mujer en este caso tiene la labor como cuidadora de enseñary educar a sus hijos e hijas con respecto a unos saberes de ambiente y dentro de estos está el no afectar al ambiente verde o natural.

- Relación Mujer-Vivo: Lo primero con lo que las mujeres relacionan lo vivo es con el ciclo de vida de los organismos (nacen, crecen, se reproducen y mueren), aunque como se sabe dentro de dicho ciclo de vida ocurre otros procesos para que lo vivo se mantenga. También se atribuye a lo vivo algunas perspectivas relacionadas con los patrones de organización que permiten la continuidad y regulación en el mundo de lo vivo como, por ejemplo, el movimiento y la respiración, y por supuesto desde la visión de lo biótico y abiótico las mujeres reconocen que dentro del mundo de lo vivo están todos los organismos, pero hacen énfasis principalmente en los animales y las plantas. Desde estas ideas, se reitera la relación de la mujer y las hembras de otras especies como dadoras, cuidadoras de la vida y también como orientadoras de la vida y los comportamientos y dinámicas dentro del ambiente. De la misma manera, las mujeres reconocen que los organismos por sí solos no se mantienen vivos, sino que necesitan de otros para mantenerse y que de este modo (hablando del mundo natural) también se estabiliza el ambiente, es decir, los organismos vivos permanecen en una interdependencia que garantiza la continuidad de la vida y esto no se lleva a cabo solo con los demás organismos sino con el ambiente y otros factores que inciden en las dinámicas del mundo de lo vivo, y para el caso de la especie humana lo vivo otorga el valor intrínseco y el carisma al poder observar, sentir y disfrutar de lo que nos rodea. También se habla de que otros animales y las plantas sirven como alimento y además aportan a la producción de oxígeno para la continuidad de la vida, y por supuesto cómo a través de lo vivo surge la medicina natural y ancestral. Una capacidad del ser humano es afectar positiva y/o negativamente el mundo de lo vivo con las acciones que se ejerzan en el mismo, y esto puede afectar las dinámicas de la vida, lo vivo y el ambiente.

- Relación Mujer-Proceso: Primero, es evidente que las mujeres acceden y son participes del Movimiento Popular de Mujeres La Sureña ya que necesitan y desean ampliar todo un campo de posibilidades dentro de la sociedad, además de ampliar y construir conocimiento con las demás mujeres, profesores y profesoras. A pesar de que ya se cuenta con esa ventaja dentro del proceso, lo que ocurre en sí mismo como proceso para y con las mujeres de la localidad de Bosa es comenzar a afianzar y reconfigurar las relaciones ya mencionadas. Las mujeres dentro del proceso establecen principalmente relaciones consigo mismas, con sus derechos y deberes. De este modo, las mujeres se reconocen y conocen, amplían su visión de sí mismas y lo que les rodea, es decir, que pueden ejercer, cumplir y proponer otros roles dentro de la sociedad. Así, se inicia todo un proceso de empoderamiento donde las mujeres configuran y construyen sociedad y dinámicas que se desarrollan dentro de la misma, que son autónomas, libres y merecen ser reconocidas y escuchadas. Afirman que saben que ser diferente a las y los demás no está mal, que la sociedad es diversa tanto el número de personas que la conforman y que de esta condición se pueden constituir nuevas formas de sociedad y conocimiento como se hace dentro del proceso. Exponen que la sociedad no es estática y que ellas pueden ser proactivas dentro del proceso de continuidad de la sociedad, son propositivas, liderezas, ya que, como se ha venido hablando, las mujeres educan y enseñan desde el hogar, para la vida, para la sociedad e influyen en la constitución de moral y valores dentro de sus familias.

Las relaciones de las mujeres con el proceso La Sureña es tan fuerte que si ellas no estuvieran presentes ni acudieran 
en cada encuentro del movimiento, este no tendría ningún sentido; las mujeres son los pies y la cabeza de este, ya que se trabaja desde, con y para ellas, y de todo lo que en este se construye, ellas se configuran a sí mismas y pueden ayudar a otras y otros.

\section{Reflexión pedagógica}

Como se ha expuesto, esta práctica pedagógica fue realizada en un ámbito educativo no formal, lo cual fue una experiencia enriquecedora como mujer y como maestra en formación. Todo el proceso fue un aprendizaje y construcción constante. Gracias a las dinámicas desde el proceso, el proyecto realizado y cada una de las mujeres del grupo de trabajo y de los demás grupos permiten el reconocimiento de sí, no solo como seres biológicos sino, como se mencionó anteriormente, como seres políticos, éticos, como mujeres activas en la sociedad, empoderadas desde el cuerpo, la cultura, la tradición, sentires y pensamientos, y desde la espiritualidad y el aprecio que se siente, se da y se recibe dentro del proceso.

Todo esto permite, sin lugar a dudas, el tejido y la construcción de saberes y para este caso, desde el área biocultural se pudo establecer un gran puente entre el conocimiento biológico, las concepciones de las mujeres y la apropiación a la cotidianidad de todo lo expuesto, dicho, constituido y aprendido.

De acuerdo con esto, es importante destacar la importancia de aprender a escuchar, ya que de esta forma se reconoce y se propicia el respeto hacia los y las demás, una acción importante en la enseñanza y la labor como maestros y maestras en este país multicultural y diverso, que cambia la forma en que se construye la sociedad desde la igualdad.

Gracias al proceso, a sus coordinadoras, maestros y maestras, y sobre todo, a cada una de las hermosas y fuertes mujeres que hicieron este proyecto posible. Gracias por permitir soñar de su mano y compartir cada experiencia siempre con una sonrisa, lo que demuestra que en esta hermosa labor, en cualquier espacio, ambiente y lugar, puede convertirse en el aula de clase, y que los procesos que allí se llevan a cabo son valiosos y contribuyen no solo a la academia sino a la vida misma.

\section{Conclusiones}

Los procesos populares son relevantes, particulares y diferentes puesto que permiten que se lleven a cabo procesos educativos, donde la enseñanza no se da entre un sujeto y un objeto o un sujeto con otro inferior para transmitir conocimiento. Estos procesos se llevan a cabo de sujeto a sujeto de una forma igualitaria, desde las relaciones y el diálogo de saberes como eje fundamental. Son espacios pensados y creados para personas y poblaciones que no son tomados en cuenta dentro de las políticas educativas y que dentro de la sociedad son vulneradas de distintas formas. Están encaminados a la conformación de un conocimiento construido y apropiado en contexto, que responda a unas necesidades y a la criticidad, y por qué no, para generar propuestas.

La educación popular con y para las mujeres permite visibilizar todo tipo de relaciones de las mujeres consigo mismas y con su ambiente, y cómo desde estas se propician espacios de y para la vida que se pueden dar desde cualquier temática y componente dentro del proceso de las mujeres, ya que ellas están vinculadas en todo momento durante cada actividad que allí se presente. Con las mujeres del grupo 2-3 en el área biocultural, a partir de la enseñanza de la Biología, se dio teniendo en cuenta las ideas previas de las mujeres, sus sentires, su historia, su lugar como fuente de vida y cuidadoras de varias formas de la misma, con el fin de vincular el saber científico con el cultural, popular y ancestral.

Desde el ecofeminismo se puede hablar desde la recuperación del principio femenino, ya que las mujeres son productoras de vida y facilitadoras de medios de subsistencia, encaminadas hacia la no jerarquización sino desde un ambiente social, cultural, político, económico igualitario para hombres y mujeres.

Es relevante el reconocimiento de sí misma como mujer con una lucha y posibilidad de empoderamiento; asimismo, se identifican a las demás desde la diversidad social, cultural, generacional y de concepciones de mundo, al generar una contraconducta hacia la educación que homogeniza el conocimiento, forma estereotipos de igualdad, etiqueta las necesidades y los propósitos de los sujetos en la sociedad anulando su identidad y visión dentro de la sociedad. Lo que permite dentro del movimiento de mujeres La Sureña es no solo enfocarse en las disciplinas que se trabajan, el enfoque de género, sino trascender en la sociedad por medio del empoderamiento de la mujer en la sociedad.

\section{Referencias}

Brito, Z. (2008). Educación Popular, Cultura e Identidad desde la perspectiva de Paulo Freire. En M. Gadotti, M. Gómez, J. Mafra, y A. Fernendes de Alencar, (Comps.), Paulo Freire. Contribuciones a la pedagogía. Buenos Aires: Clacso. 
Cerda, H. (1991). Capítulo 7. Medios, instrumentos, técnicas y métodos en la recolección de datos e información. Recuperado de http://postgrado.una. edu.ve/metodologia2/paginas/cerda7.pdf

Colunga, S., García, J., y Blanco, C. (2013). El docente como investigador y transformador de sus propias prácticas. La investigación-acción en educación. Transformación, 9(1), 14-23.

Perez, G. (1994). Investigación cualitativa: retos e interrogantes. Madrid: Editorial Muralla S.A.

Sáenz, I., y Rapacci, L. (2013). La Educación Popular Feminista: Una perspectiva que se consolida. Red de Educación Popular Entre Mujeres de Latinoamérica y El Caribe (REPEM). Recuperado de http://
www.democraciaycooperacion.net/IMG/pdf/180b39_f0ca0085a6f98545c61b86109f0bf942.pdf

Taylor, S. J., y Bogdan, R. (1987). Introducción a los métodos cualitativos. Barcelona: Paidós.

Torres, S., González, C., y Pérez, L. (2012). Propuesta de Educación de Adultas con Enfoque de Género. Bogotá: Movimiento Popular de Mujeres La Sureña. Documento Institucional.

Urbina, F. (2013). Notas para un "Diálogo de Saberes". Recuperado de http://www.artesaniasdecolombia. com.co:8080/Documentos/Contenido/9067_notas_para_un_dialogo_de_saberes.pdf 